\title{
List of Reviewers 2021
}

Thanks to our reviewers and corresponding editors!

The Journal of Pediatric Rehabilitation Medicine would like to recognize the following for their time and commitment in providing scientific reviews from November 1, 2020, through October 31, 2021.

Sarah Afzal

Rishi Agrawal

Unoma Akamagwuna

Michele Alaniz

Leland Albright

Kristen Allison

Andrew Althouse

Kirk Anderson

Marcella Andrews

Frieda Ansoanuur

Susan Apkon

Kristian Aquilina

Mokhtar Arazpour

Afua Asante

Christine Assaiante

Rita Ayyangar

Shasha Bai

Gandhi Balaji

Lidija Barbarić

Diane Barsky

Jules Becher

Melanie Bell

Melissa Bellin

David Berbrayer

Matteo Bertucco

Catherine Billups

Eve Blair

Carol Boliek

Jael Bootsma

Glendaliz Bosques

Elizabeth Boyer

Joline Brandenburg

Reinald Brunner

Cathleen Buckon

Samantha Butler

Rhonda Cady

Bo Cai

Chantal Camden

Kristen Campbell

Freddy Cao

Terri Carry

Heidi Castillo
Jonathan Castillo

Uday Chalwadi

Kate Chang

Alexia Charpentier

Lorry Chen

Earl Cheng

Yin-Hsiu Chien

Erika Cloodt

Andrew Collins

Keith Cordner

John Costello

Colleen Coulter

Giovanni Cucchiaro

Afsaneh Dadarkhah

Noémi Dahan-Oliel

Diane Damiano

Loren Davidson

Kimberly Davis

Shay Dawson

Steven Day

María de los Ángeles Dallo

Stephanie DeLuca

Anton Dietzen

Cynthia Dodds

Nienke Dosa

Colleen Driscoll

Helene Dumas

Stacey Dusing

Rochelle Dy

Meta Eek

Joseph Egger

Murat Ersoz

Nathan Evanson

Moretto Fabien

Aishah Fauzi

Andrea Fergus

Susan Fernandes

Jonathan Finder

Ericka Fink

Mark Fisher

Alyssa Fiss

Jack Fletcher 
Janet Foote

Karen Fratantoni

Ellen Fremion

John Frino

Jack Fu

Molly Fuentes

Ellen Fung

Shruti Gadkari

Deborah Gaebler-Spira

Charlene Gaebler-Uhing

Ewa Gajewska

Sandra Galbally

Paula Geigle

Reint Geuze

Esra Giray

Vithya Gnanakumar

Daniela Godoi Jacomassi

Keith Goldfeld

Jay Goldsmith

Grace Gombolay

Wendy Goodwin

Caroline Gormley

Rik Gosselink

Kerr Graham

Susan Gray

Mike Green

Renata Guare Romano

Mintaze Günel

Yian Guo

Juliet Haarbauer-Krupa

Zehra Habib Hasan

Stacey Hall

Dan Halvorsen

Deanna Hansen

Celia Harding

Kimberly Hartman

Caroline Hastings

Matt Hayat

Katherine Hayes

Jill Heathcock

Monica Hedenbro

Florian Heinen

Lydia Henderson

Kinsey Herrin

Nazanin Heydarian

Scott Hoffinger

Betsy Hopson

Brittany Hornby

Saskia Houwen

Chia-Wei Hsu

John Hughes
Andrew Hull

Lou Hunter

Jane Hutton

Susan Iannaccone

Katarzyna Ibanez

Didem Inanoglu

Janet Jackson-Coty

Alireza Jafari

Jessica Jarvis

Ira Jeglinsky

Dorothee Jelsma

Kristin Jensen

Abigail Johnson

Harrison Jones

Christopher Joseph

David Joseph

Nadja Kadom

Turhan Kahraman

John Kairalla

JoAnne Kanas

Kyra Kane

Mitul Kapadia

Natalie Karavarsamis

Benjamin Katholi

Walter Kaufmann

Heidi Kecskemethy

Jennifer Keller

Kiersten Kelly

Maryellen Kelly

Ann Kennedy-Behr

Kyla Kent

Atif Khan

Edward Kim

Heakyung Kim

Ryutaro Kira

Virginia Knox

Fatmanur Aybala Koçak

Kat Kolaski

Lucy Kompaniyets

Christine Koterba

Linda Krach

Jessica Kramer

Breanna Krueger

Sally Le Cras

Martin Lemay

Traci Leong

Brenda Lessen Knoll

Enoch Leung

Jared Levin

Todd Levy

Chen Li 
Nan Li

Yimei Li

Cornelia Lieb-Lundell

Siyao Liu

Wei Liu

Roslyn Livingstone

Ji Meng Loh

Helen Long

Christopher Lunsford

Lívia Magalhães

Zoe Mailloux

Arnab Maity

Joyce Maring

Georgios Markozannes

Petra Marsico

Brendan Martin

Gaby Martinez

Andrea Martinuzzi

John McGuire

Matthew McLaughlin

Kelly McNally

Jill Meilahn

André Meireles

Michelle Melicosta

Anton Miller

Sedigheh Mirzaei Salehabadi

Masyitah Mohammad Khayat

Shoubhik Mondal

Jake Mooney

Eleanor Morris

Alana Moser

Theresa Moulton

Sue Mukherjee

Noureddin Nakhostin Ansari

Matthew Nedjat-Haiem

Ginny Nelson

Samuel Nemanich

Janet Neufeld

Rachel Neufeld

Pengsheng Ni

Karen Nielsen

Dejan Nikolić

Donna Nimec

Iona Novak

Marykay Nutini

Kevin O'Brien

Roberta O'Shea

Rory O'Sullivan

James Ogilvie

Tatiana Ogourtsova

Jaclyn Omura
Pornsuree Onmanee Kuvijitsuwan

Arve Opheim

Maria Chiara Oprandi

Elaine Owen

Yi-Tsen Pan

Teresa Paolucci

Shrutika Parab

Michael Partington

Mario Petersen

Paula Peterson

Adolf Pfefferbaum

Kelly Pham

Elaine Pico

Stephanie Plamondon

Annie Pouliot-Laforte

Luke Prendergast

David Pruitt

Amy Rabatin

Eugene Rameckers

Sharon Ramey

Diann Reischman

Nushka Remec

Monique Ridosh

Andy Ries

Elias Rizk

William Rizzo

Desiree Roge

Lynne Romeiser-Logan

Sam Rosenfeld

Sandy Ross

David Roye

A.J. Rush

Ji Hoon Ryoo

Cristina Sadowsky

Kashika Sahay

Ghazala Saleem

Kathleen Sawin

Susan Sawyer

Phoebe Scott-Wyard

Julieanne Sees

Donald Sharpe

Eileen Sherburne

David Sherry

Maurice Sholas

Susan Sienko

Beth Slomine

Lynette Smith

Patrick Spicer

Deo Kumar Srivastava

Katy Stauffer

Julie Stebbins 
Richard Stevenson

Jon Stone

Alan Stotts

Renat Sukhov

Stacy Suskauer

Michael Sussman

Hillary Swann-Thomsen

George Tharion

Ryan Theis

Judy Thibadeau

Sruthi Thomas

Debbie Thorpe

Ann Tilton

Rick Tivis

Jonathan Tolentino

Margaret Turk

Marietta van der Linden

Huub van Hedel

Jilda Vargus-Adams

Christian Vercler

Anna Vergun

Gijs Verkerk

Joshua Vova

Mladenka Vukojević

Hui-Yi Wang
Yan Wang

Seth Warschausky

R Scott Watson

Frances Weaver

Erin Wentzell

John Wiener

Kayla Williams

Sian Williams

Ruth Williams-Hooker

Pam Wilson

Hadley Wood

Ed Wright

Colleen Wunderlich

Rui Xiao

Abdulsalam Yakasai

Michele Yang

Gayane Yenokyan

Heather Young

Michele Zaccario

Magy Zahran

Qianzi Zhang

Yunxi Zhang

Xiwen Zhao

Hussein Ziab

Jill Zwicker 